\title{
EVALUATION OF EFFICIENCY OF LOW-LEVEL LASER ON RELAPSE AFTER ORTHODONTIC TREATMENT: A RANDOMIZED CONTROLLED CLINICAL TRIAL
}

\author{
Ousama Abo Ayach', Rania Hadad', Omar Hamadah² \\ 'Department of Orthodontics and Dentofacial Orthopedics, Faculty of Dental Medicine, Damascus University, Syria \\ ${ }^{2}$ Department of Oral Medicine, Faculty of Dental Medicine, Damascus University, Syria
}

\begin{abstract}
INTRODUCTION: The retention is a critical stage after the active orthodontic treatment which has been always aimed at being shorter and more effective and acceptable.

OвJECTIVEs: The purpose of this study was to evaluate the effectiveness of 3 protocols of fixed and removable retainers, and to investigate the effect of low-level laser (LLL) on the amount of relapse and the duration of the retention phase.

MATERIAL AND METHODS: A total of 54 straightening and alignment patients' casts and radiographs as well as debond of orthodontic appliances and 1-year recall were evaluated using the American Board of Orthodontics $(\mathrm{ABO})$ discrepancy index. These patients were equally divided into 3 retention protocol groups, including upper and lower bonded retainers (BRs), upper and lower vacuum-formed retainers (VFRs), and upper and lower VFRs with LLL application. A continuous wavelength (Ga-Al-As) semi-conductor laser was applied in the third group, with an $808 \mathrm{~nm}$ wavelength of special protocol.

RESULTS: Only VFR LLL group improved in total cast radiograph evaluation (CRE) scores, while in the other two groups, total CRE scores decreased insignificantly for BRs and significantly for VFRs. At the end of observation period, lowest rate of relapse was noted among the patients of laser group, followed by BRs and VFRs groups, respectively. The average time required to reach 12 hours of retainer's use in laser group was 8.33 weeks, whereas the required time to reach intermittent use was 25 weeks.

ConCLUsions: During retention phase, only buccolingual inclination among CRE variables improved significantly. While alignment, overjet, interproximal contact, and total CRE worsened significantly, changes in marginal ridges, occlusal contacts, occlusal relationship, and root angulation were insignificant. Both BRs and VFRs are effective methods to prevent a relapse, with clinically insignificant post-treatment changes. Applying LLL with VFRs would significantly reduce the number of relapses, enhance the total CRE as well as decrease the required time of retainers' wearing and overall retention phase.
\end{abstract}

KEY wORDs: randomized controlled clinical trial, relapse, low-level laser, ABO objective grading system, vacuumformed retainers.

J Stoma 2021; 74, 3: 140-146

DOI: https://doi.org/10.5114/jos.2021.108838

JOURNAL OF STOMATOLOGY CZASOPISMO STOMATOLOGICZNE

AdDResS FOR CORRESPONDENCE: Dr. Ousama Abo Ayach, Department of Orthodontics and Dentofacial Orthopedics, Faculty of Dental Medicine, Damascus University, Mazze highway, Damascus, Syria, P.O. Box 9006, e-mail: abouayash.ouss@gmail.com

ReCEIVED: 20.01.2021 • ACCEPTED: 24.05.2020 • Published: 30.08.2021 


\section{INTRODUCTION}

Maintaining the teeth in optimal aesthetic and functional positions after treatment is the most used definition of "retention" that is applied in almost every patient [1]. Despite widespread use of retainers as an essential post-treatment phase, clinicians have not come to a consensus about the necessity of retention, which remains a controversial subject among orthodontists [2, 3].

Although there is a growing trend towards more fixed retention, and so called "invisible retainers" are more used instead of Hawley appliances, the choice of type of orthodontic retainer seems to be mainly based on experience of an orthodontist [4-6]. According to Littlewood et al's systematic review, this uncertainty occurs due to large gaps in the literature, providing no evidence of superiority of fixed over removable retainers (or vice versa) in terms of their effectiveness, in addition to limited evidence concerning associated harms, longterm implications, and patient's compliance [7].

Another dilemma in the orthodontic practice is the required time of retainer's wear as well as the recommended duration of retention phase [8]. Potential complications associated with prolonged retention, including the periodontium and patient's compliance, create a uncertainty regarding the need of long-lasting, and indeed, indefinite retention as a long-term experience [9]. The tendency to rapidly reverse orthodontic results has encouraged researches to explore ways to reduce or prevent unwanted movements. A number of pharmacological agents were investigated to reduce the relapse, includinglocal or systemic applications in experimental animals, and some of these factors were found effective [10-15]. Outcomes of these factors varied, but in general, the relapse was alleviated by tissue remodeling around the teeth. Therefore, it is possible to rely on any mechanism that is capable of altering a relapse's natural process as a support of the retention stage.

Low-level laser therapy (LLLT) has been widely used in dentistry; as non-invasive technique, it is able to produce bio-revitalization effects and thus can be used in the field of stabilizing orthodontic treatment results [16].

In a study, Goulart et al. [17] found that LLL in specific conditions and doses can slow orthodontic movement and therefore, it can be used to prevent a relapse by accelerating the osteogenesis $[18,19]$.

By reviewing previous research, despite a small amount of clinical studies investigating the effect of LLLT on acceleration of dental movement, there is no study on its effect on recurrence of dental movement after orthodontic treatment. Nevertheless, several studies were examining this issue on animals, and all confirmed the positive effect of LLL on relapse reduction [20-22]. Therefore, the current study aimed to evaluate the effectiveness of 3 protocols of fixed and removable retainers as well as to investigate the effects of low-level laser on the number of relapse and the total duration of retention phase.

\section{MATERIAL AND METHODS}

This was a 3-arm parallel group, randomized controlled clinical trial, with a $1: 1: 1$ allocation ratio. The study was ethics committee-approved by the Council of Higher Education and Scientific Research of Damascus University (Ref. No., 5100/2018), and informed consents were obtained from all participants.

The study population included 54 patients $(29 \mathrm{fe}$ males and 25 males; between 18 and 26 years of age; mean age, $20.5 \pm 1.8$ years) who were randomly selected from the Department of Orthodontics and Dentofacial Orthopedics, Faculty of Dental Medicine, Damascus University. All subjects included in this study had to fulfil the following criteria: 1) moderate crowding (4-6 mm) and skeletal angle class $\mathrm{I}$, treated by straightening and alignment without extraction; 2) underwent a successful orthodontic treatment according to the $\mathrm{ABO}$ rating system, with less than 20 points; 3) showing with normal growth pattern, normal functional examination, and permanent and complete occlusion; 4) presenting with healthy supportive tissues, good oral hygiene, without absorption in the roots of any of the studied teeth; 5) no previous history of trauma, endodontic treatments, general or local disease, syndromes, or facial and jaw disorders.

Study sample size was determined based on primary objective of comparing the efficacy of each retainer group in preventing the relapse in 1-year post-orthodontic retention. Assuming a change of $0.5 \mathrm{~mm}$ as the least clinically significant difference, a common standard deviation of $0.5 \mathrm{~mm}$, a study power of $90 \%$, and a significance level of $5 \%$, each study group required 18 subjects.

Having consented to participate in the present study, the subjects were randomly allocated into either upper or lower bonded canine retainers (BRs), vacuum-formed retainers (VFRs), or VFRs with low-level laser (LLL) application. Depending on a computerized randomization program, sequentially numbered, opaque, and sealed envelopes were prepared in advance by an independent person.

After the patients' allocation, and due to the nature of intervention, it was not possible to blind the patients or researchers in this study. Blinding of the outcome assessor investigating the stability was undertaken by replacing the patient's information on the casts and radiographs by a 4-digit code.

Bonded retainers (BRs) were prepared using a 0.0195 in $(0.45 \mathrm{~mm})$ 3-stranded twist flex stainless steel wire (Wildcat; GAC International, Bohemia, New York, USA). The wire was shaped by the researcher against the dental casts to passively lie beside lingual surfaces of the upper and lower incisors and canines. The wire was not contoured interproximally. The wire was bonded using a low viscosity light-cured composite (Transbond $^{\mathrm{Tm}}$ LV; 3M Unitek, Monrovia, California, USA), following separate application of etchant (37\% phosphoric 
acid) and primer (Transbond ${ }^{\mathrm{TM}} \mathrm{XT}$ adhesive primer; 3M Unitek). Any excess bonding substance in contact with the gingival tissues was carefully removed. VFRs $\left(\right.$ Essix $^{\mathrm{mm}} \mathrm{C}+$ ) were formed for both groups of removable retainers using Essix $^{\text {Tm }}$ device. Retainers were made of $1 \mathrm{~mm}$ thick, hard, thermoplastic vacuum sheets by standard characteristics for all patients, so that they could cover occlusal surfaces of all erupting teeth and extend over 3-4 mm from gingival margins [23, 24].

The participants in the VFRs only group were instructed to wear their retainers full time for six months, followed by six months of overnight wear, and last six months of intermittent nocturnal use, which is the most commonly used retention protocol [25]. The laser group participants were requested to full time wear their retainers for one month, and then reduce the time of wearing by three hours weekly in second month, so that at the end of that month, only night-time wear was required. This reduction was used only if the patient felt no pressure of the retainer, remained neutral to the teeth, and under weekly supervision of the researcher.

At the time of retainer application, both written and verbal oral hygiene instructions were given, including methods for interdental cleaning around BRs.

A continuous $808 \mathrm{~nm}$ wavelength with 4 Joules per point power and 15 seconds per point were applied using Klas-DX laser 808 low-level laser device (Konflec Corporation), at the Laser Research Unit, Faculty of Dental Medicine, Damascus University. This is a semi-conductor laser, with gallium aluminum arsenide ( $\mathrm{Ga}-\mathrm{Al}-\mathrm{As}$ ) as the laser medium.

The laser was applied on the root of each of the upper and lower teeth in four points, where the tooth root would theoretically be divided into two halves (cervical and apical) and the head of the device was placed at the buccal side of the root toward the center of each half, in contact with oral mucosa and perpendicularly to the root axis [26]. The laser was re-applied in the same way at the palatal side of the root. The total application

TABLE 1. Differences in each component of the American Board of Orthodontics objective grading system (ABO-OGS) in the whole sample

\begin{tabular}{|l|c|c|c|}
\hline Variable & Mean at T0 & Mean at T2 & \multicolumn{1}{c|}{$\boldsymbol{p}$-value } \\
\hline Alignment & 0.389 & 1.000 & $<0.0001^{*}$ \\
\hline Marginal ridges & 0.722 & 0.740 & 0.878 \\
\hline Buccolingual inclination & 0.981 & 0.667 & $0.008^{*}$ \\
\hline Occlusal contacts & 1.037 & 0.852 & 0.296 \\
\hline Occlusal relationship & 0.981 & 1.129 & 0.132 \\
\hline Overjet & 0.370 & 0.556 & $0.017^{*}$ \\
\hline Interproximal contacts & 0.259 & 0.648 & $0.002^{*}$ \\
\hline Roots angulation & 0.889 & 0.907 & 0.569 \\
\hline Total & 5.629 & 6.500 & $0.033^{*}$ \\
\hline *Statistically significant. & & &
\end{tabular}

time was 60 seconds per tooth. The laser was used on the first day of VFRs application, and then on the $3^{\text {rd }}, 7^{\text {th }}$, and $14^{\text {th }}$ day of the first month. Starting from the second month, it was applied every two weeks, until the patient reached the intermittent wear phase [26].

The primary outcome of the study was to evaluate the clinical effectiveness of the three retainer types in preventing post-treatment changes over the initial 12-months of retention. Post-treatment changes were defined by evaluating the casts using the ABO discrepancy index [27]. Alginate impressions were obtained and measured by the same examiner at three points during the study (T0-T2): T0 at the orthodontic appliances' debonding, T1 after six months, and T2 after a year of beginning of retention phase.

Alignment/ rotation, marginal ridges, buccolingual inclination, overjet, occlusal contacts, occlusal relationship, interproximal contacts, and root angulation were the variables evaluated using cast radiograph evaluation (CRE).

Paired $t$-test was used to compare paired sample means. Analysis of variance tests were applied to compare the means for more than 2 groups. A two-sided 0.05 a level defined a statistical significance.

\section{RESULTS}

To identify the overall differences in each element of the American Board of Orthodontics objective grading system (ABO-OGS) during the retention phase irrespective of the type of retainer, paired sample $t$-test was used to compare the change of variables in all 54 subjects. The differences in alignment/rotation, buccolingual inclination, overjet, interproximal contacts, and total CRE score showed statistical significance from debonding to 1-year recall. However, the differences in marginal ridges, occlusal contacts, occlusal relationship, and root angulation were observed with no statistically significant differences in the same period of observation.

While the buccolingual inclination and the occlusal contacts were the only improved variables, the worsening was shown in all the other variables.

In total, a worsening of 0.9 point was proved from debonding to 1-year recall as the total CRE increased from 5.6 to 6.5 points in this period of time (Table 1 ). The comparison of changes in variables in different retention protocols is presented in Table 2. There were statistical differences for alignment/rotation, buccolingual inclination, marginal ridges, occlusal contacts, and interproximal contacts in different retention groups. In contrast, there was no statistical difference between retention protocols for overjet, occlusal relationship, and root angulation. The change of total CRE score from debonding to recall differed significantly among the 3 retention groups (Table 2).

Table 3 shows the comparison between the variables' means of each retention group at the beginning 
TABLE 2. Comparison of the variables' changes in different retention protocols

\begin{tabular}{|c|c|c|}
\hline Variable/Retention group & $\begin{array}{c}\text { Mean of difference } \\
\text { (T2-T1) }\end{array}$ & $p$-value \\
\hline \multicolumn{3}{|l|}{ Alignment } \\
\hline BR & -1.500 & \multirow[t]{3}{*}{$<0.0001^{*}$} \\
\hline VFR & -0.045 & \\
\hline Laser & -0.056 & \\
\hline \multicolumn{3}{|l|}{ Marginal ridges } \\
\hline BR & 0.222 & \multirow[t]{3}{*}{$0.038^{*}$} \\
\hline VFR & -0.500 & \\
\hline Laser & 0.167 & \\
\hline \multicolumn{3}{|l|}{ Buccolingual inclination } \\
\hline BR & 0.722 & \multirow[t]{3}{*}{$<0.0001^{*}$} \\
\hline VFR & -0.272 & \\
\hline Laser & 0.555 & \\
\hline \multicolumn{3}{|l|}{ Occlusal contacts } \\
\hline BR & 0.833 & \multirow[t]{3}{*}{$<0.0001^{*}$} \\
\hline VFR & -1.000 & \\
\hline Laser & 1.056 & \\
\hline \multicolumn{3}{|l|}{ Occlusal relationship } \\
\hline BR & -0.222 & \multirow[t]{3}{*}{0.053} \\
\hline VFR & -0.375 & \\
\hline Laser & 0.167 & \\
\hline \multicolumn{3}{|l|}{ Overjet } \\
\hline BR & -0.222 & \multirow[t]{3}{*}{0.462} \\
\hline VFR & -0.045 & \\
\hline Laser & -0.056 & \\
\hline \multicolumn{3}{|l|}{ Interproximal contacts } \\
\hline BR & -0.833 & \multirow[t]{3}{*}{$0.021^{*}$} \\
\hline VFR & -0.091 & \\
\hline Laser & -0.222 & \\
\hline \multicolumn{3}{|l|}{ Roots angulation } \\
\hline BR & -0.055 & \multirow[t]{3}{*}{0.273} \\
\hline VFR & 0.409 & \\
\hline Laser & 0.056 & \\
\hline \multicolumn{3}{|l|}{ Total } \\
\hline BR & -1.056 & \multirow[t]{3}{*}{$<0.0001^{*}$} \\
\hline VFR & -3.250 & \\
\hline Laser & 1.667 & \\
\hline
\end{tabular}

and the end of the observation period. Only the VFRs LLL group improved in the total CRE score, and this group demonstrated a statistical improvement in 2 variables. The bonded retainer cohort had 2 variables with statistical improvements, but 2 other variables revealed
TABLE 3. Comparison of the variables' means of each retention group at the beginning and the end of observation period

Retention group/Variable Mean at T0 Mean at T2 $p$-value BR

\begin{tabular}{l|c|c|c}
\hline Alignment & 0.222 & 1.722 & $<0.0001^{*}$ \\
\hline Marginal ridges & 0.889 & 0.667 & 0.331 \\
\hline Buccolingual inclination & 1.000 & 0.278 & $0.002^{*}$ \\
\hline Occlusal contacts & 1.278 & 0.444 & $0.001^{*}$ \\
\hline Occlusal relationship & 1.056 & 1.278 & 0.260 \\
\hline Overjet & 0.333 & 0.556 & 0.260 \\
\hline Interproximal contacts & 0.278 & 1.111 & $0.007^{*}$ \\
\hline Roots angulation & 0.889 & 0.944 & 0.331 \\
\hline Total & 5.944 & 7.000 & 0.130
\end{tabular}

VFR

\begin{tabular}{l|c|c|c|}
\hline Alignment & 0.444 & 1.000 & $0.001^{*}$ \\
\hline Marginal ridges & 0.555 & 0.722 & 0.507 \\
\hline Buccolingual inclination & 1.000 & 1.000 & 1.000 \\
\hline Occlusal contacts & 0.389 & 1.333 & $<0.0001^{*}$ \\
\hline Occlusal relationship & 0.667 & 1.722 & $0.002^{*}$ \\
\hline Overjet & 0.500 & 1.056 & $0.001^{*}$ \\
\hline Interproximal contacts & 0.333 & 0.778 & 0.119 \\
\hline Roots angulation & 0.833 & 0.444 & 0.110 \\
\hline Total & 4.722 & 7.944 & $<0.0001^{*}$
\end{tabular}

Laser

\begin{tabular}{|c|c|c|c|}
\hline Alignment & 0.500 & 0.556 & 0.668 \\
\hline Marginal ridges & 0.722 & 0.556 & 0.421 \\
\hline Buccolingual inclination & 0.944 & 0.389 & $0.008^{*}$ \\
\hline Occlusal contacts & 1.444 & 0.389 & $<0.0001^{*}$ \\
\hline Occlusal relationship & 1.222 & 1.056 & 0.187 \\
\hline Overjet & 0.278 & 0.333 & 0.331 \\
\hline Interproximal contacts & 0.167 & 0.389 & 0.163 \\
\hline Roots angulation & 0.944 & 0.889 & 0.331 \\
\hline Total & 6.222 & 4.556 & $<0.0001^{*}$ \\
\hline
\end{tabular}

statistical worsening, and the overall CRE change was insignificant. Four variables demonstrated statistically significant worsening in the VFRs group. This cohort demonstrated the least improvement and biggest number of relapses among the 3 retention groups.

The average time required to reach 12 hours of retainer's use in the laser group was 8.33 weeks, which was approximately one-third of the required 24 weeks in the conventional protocol. The required time to reach the intermittent wear use was 25 weeks, which was approximately half of the 52 weeks required in the standard protocol (Table 4). 
TABLE 4. Average time required to reach 12 hours and intermittent use of retainer

\begin{tabular}{|l|c|c|c|c|c|}
\hline & Lower & Upper & Mean & SD \\
\hline Average time required to reach 12 hours & 8.00 & 11.00 & 8.333 & 0.840 \\
\hline Average time required to reach intermittent use & 24.00 & 30.00 & 25.278 & 1.934 \\
\hline
\end{tabular}

\section{DISCUSSION}

In the literature, different methods were used to evaluate the number of relapses. Even though, Little index was used in the study of Forde et al. [28], the majority of studies used a group of casts' analyzes, with inter-canines width, inter-molars width, length of dental arch, overjet, and overbite [29-31]. In the present study, the $\mathrm{ABO}$ objective indicator was used to compare the number of changes in occlusions in different retention protocols. This indicator is widely applied in studies investigating treatments' effectiveness, and it is based on eight quantitative variables evaluation. Moreover, it is more comprehensive and, unlike other methods, it can reveal a positive change, which is equivalent to an improvement in the occlusion during retention phase [32].

In the present study, a detailed analysis of the components of CRE scores for the whole sample showed that some variables demonstrated an improvement with lower CRE scores at 1 year after the debonding date, including the buccolingual inclination and occlusal contacts. The greatest improvement of CRE score was about 0.3 point in the buccolingual inclination average. In the contrary, an increase could be observed in the alignment/rotation, overjet, and total CRE scores (statistically significant) as well as in the marginal ridges, occlusal relationship, and root angulation (no statistical significance, which can be interpreted as no real change). These changes were interpreted to be signs of relapses or unfavorable tooth movements. The greatest worsening was noted in the alignment/rotation variable, with approximately 0.62 point of change. These settling changes should be considered in the decision to remove orthodontic appliances, as the overall anterior and posterior positions as well as relations of the teeth would not improve during settling phase.

In the present study, the improvement of occlusal contacts coincides with previous research [33-35]. Contrary to their findings and on the base of the current study results, it is not advisable for clinicians to neglect the use of occlusal equilibration of cusp tips on the day of debonding, expecting an improvement in the occlusion over a year of settling, since that improvement was questionable.

According to the findings of this study, a less favorable and stable occlusion 1 year after the debonding date can be expected. The average CRE score for the whole study cohort worsened in average by 0.9 point from 5.6 at debond to 6.5 after 1 year of the retention phase.
The maximum score of the ABO-OGS for any orthodontic case to be successful according to the $\mathrm{ABO}$ is 27 points. On average, all cases included in this study met the board certification standards on the day of debonding and after 1 year of settling, which can mean that the worsening was not clinically significant, and the three retention protocols were effective in preserving orthodontic treatments' outcomes. The results from this study coincide with those obtained from a study by Greco et al. [36], which showed a worsening in the overall CRE score after the retention period.

On the other hand, these results differed from those of two other studies [32,37], in which the overall CRE score improved after the retention period. However, the results of these three above-mentioned studies were comparable regarding worsening of alignment, improving of buccolingual inclination, and occlusal contacts during the retention period.

In the present study, by comparing the results of total CRE values between the three retention groups at T0, there were no statistically significant differences, which means that the results of orthodontic treatments were close in the three groups to exclude any effect of the initial malocclusion on the degree of relapse among the investigated groups.

The results of the ABO index within the same group between the study times showed that (although the total CRE scores worsened in both the BRs and VFRs groups) they remained around 7 points, meaning that both methods were effective in stabilizing the results of orthodontic treatments, and this was consistent with most of the previous studies [31].

The worsening in occlusion that was significant only in the VFRs group, contradicts with results of a study by Hoybjerg et al. [32], which compared the change of $\mathrm{ABO}$ index using three retention protocols, including BRs and VFRs. They found that the occlusion improved with a decrease in the value of index after one-year observation. This difference can be attributed to the fact that the average value of total CRE in Hoybjerg's study was significantly higher, ranging between 27 and 29 points at T0. However, it needs to be considered that their study included patients with and without extractions, which could strongly impact the results.

Additionally, the current study demonstrated that the overall CRE in the laser group decreased significantly during the first year of retention, which indicates an improvement of the occlusion during this period. This result cannot be compared with previous studies because 
there are no clinical researches investigating the effect of low-level laser on the occlusal changes during retention stage.

At the end of the observation period, the lowest rate of relapse was among the patients in the laser group, followed by the BRs and VFRs groups. These results were consistent with many previously published studies $[9,28$, 38], demonstrating BRs as more effective in resisting relapses than VFRs.

Also, the results of the current study agree with the results of a systematic review of Giudice et al. [31] as well as with the results of all studies in the medical literature comparing different retention methods confirming BRs as more effective in preventing relapses than removable appliances.

On the other hand, the results of this study differ from those of O'Rourke's [25], who observed that relapse rate (although it was lower in BRs group after 6 months of retention) became equal to that of VFRs group after one year. This difference can be attributed to the fact that O'Rourke investigated relapse on the lower jaw only and therefore, did not consider occlusal changes.

The results of the current research also differ with yet another study [39], which compared the effectiveness of BRs, Hawley device with partial wear, and VFRs with full-time wear. The effectiveness of these three protocols was equal after one year of observation, and this difference could be attributed to small sample size [39].

The retention protocol was adopted in the laser group by starting with a four-week stage of full-time wearing to ensure adequate retention was obtained and a quick relapse during this period was prevented. The time of use was gradually reduced by three hours per week, if the neutrality condition of the retainer was met, depending on the researcher's careful examination, and the patient's sensing of the pressure applied by the appliance. The protocol used enabled the determination of required time to wear the retainer without exposing the patient to the risk of a relapse.

The average period required to reach the 12-hour wearing time in the laser group was about two months, which was one third of the six months needed in the conventional retention protocol. Additionally, all patients were able to follow the intermittent use of VFRs after thirty weeks of retention. Therefore, the number of hours required to wear VFRs during the day, in addition to the total duration of the retention phase, was statistically and clinically significantly reduced. Unfortunately, these results could not be compared with those from the literature due to lack of clinical studies investigating the effect of LLL on the duration of the retention stage.

\section{CONCLUSIONS}

With the limitations of the current study, it could be concluded that: 1 . During the 1 -year of settling phase in both of fixed and removable retainers, only the buccolingual inclination among CRE variables improved significantly. While the alignment, overjet, interproximal contact, and total CRE worsened significantly, the changes in marginal ridges, occlusal contacts, occlusal relationships, and root angulation were insignificant; 2. At the end of the 1-year observation, the BRs, and VFRs retention methods showed a worsening of CRE scores. However, those changes were clinically insignificant, and both methods were effective in stabilizing the results of orthodontic treatments; 3. Using lowlevel laser (LLL) with VFRs could significantly decrease the relapse level and enhance the overall CRE at the end of observation period; 4. Applying LLL with VFRs may significantly decrease the required time of retainers' wearing and the overall retention phase.

\section{CONFLICT OF INTEREST}

The authors declare no potential conflicts of interest with respect to the research, authorship, and/or publication of this article.

\section{References}

1. Bearn DR. Bonded orthodontic retainers: a review. Am J Orthod Dentofacial Orthop 1995; 108: 207-213.

2. Dosanjh MSK. Efficacy of different retention types post-orthodontic treatment. Master's theses. University of Connecticut Graduate School 2011.

3. Kingsley NW. A treatise on oral deformities as a branch of mechanical surgery. New York: D. Appleton; 1880.

4. Keim RG, Gottlieb EL, Vogels DS 3rd, Vogels PB. 2014 JCO study of orthodontic diagnosis and treatment procedures, Part 1: results and trends. J Clin Orthod 2014; 48: 607-630.

5. Pratt MC, Kluemper GT, Hartsfield JK Jr, Fardo D, Nash DA. Evaluation of retention protocols among members of the American Association of Orthodontists in the United States. Am J Orthod Dentofacial Orthop 2011; 140: 520-526.

6. Meade MJ, Millett D. Retention protocols and use of vacuumformed retainers among specialist orthodontists. J Orthodont 2013; 40: 318-325.

7. Littlewood SJ, Millett DT, Doubleday B, Bearn DR, Worthington HV. Retention procedures for stabilising tooth position after treatment with orthodontic braces. Cochrane Database Syst Rev 2016; 2016: CD002283.

8. Valiathan M, Hughes E. Results of a survey-based study to identify common retention practices in the United States. Am J Orthod Dentofacial Orthop 2010; 137: 170-177.

9. Al Moghrabi D. Orthodontic retention: a prospective evaluation of stability. A thesis submitted for the degree of Doctor of Philosophy. Centre for Oral Bioengineering Barts and The London School of Medicine and Dentistry. Queen Mary University of London; 2019.

10. Kim TW, Yoshida Y, Yokoya K, Sasaki T. An ultrastructural study of the effects of bisphosphonate administration on osteoclastic bone resorption during relapse of experimentally moved rat molars. Am J Orthod Dentofacial Orthop 1999; 115: 645-653.

11. Zhao N, Lin J, Kanzaki H, et al. Local osteoprotegerin gene transfer inhibits relapse of orthodontic tooth movement. Am J Orthod Dentofacial Orthop 2012; 141: 30-40.

12. Hudson JB, Hatch N, Hayami T, et al. Local delivery of recombinant osteoprotegerin enhances postorthodontic tooth stability. Calcif Tissue Int 2012; 90: 330-342. 
13. Han G, Chen Y, Hou J, et al. Effects of simvastatin on relapse and remodeling of periodontal tissues after tooth movement in rats. Am J Orthod Dentofacial Orthop 2010; 138: 550.e1-e7.

14. Hirate Y, Yamaguchi M, Kasai K. Effects of relaxin on relapse and periodontal tissue remodeling after experimental tooth movement in rats. Connect Tissue Res 2012; 53: 207-219.

15. Hassan AH, Al-Hubail A, Al-Fraidi AA. Bone inductive proteins to enhance postorthodontic stability: a pilot study. Angle Orthod 2010; 80: 1051-1060.

16. Torri S, Weber JBB. Influence of low-level laser therapy on the rate of orthodontic movement: a literature review. Photomed Laser Surg 2013; 31: 411-421.

17. Goulart CS, Nouer PRA, Mouramartins L, Garbin IU, Lizarelli RDFZ. Photoradiation and orthodontic movement: experimental study with canines. Photomed Laser Ther 2006; 24: 192-196.

18. Zahra SE, Elkasi AA, Eldin MS, Vandevska-Radunovic V. The effect of low level laser therapy (LLLT) on bone remodelling after median diastema closure: a one year and half follow-up study. Orthodontic Waves 2009; 68: 116-122.

19. Saito S, Shimizu N. Stimulatory effects of low-power laser irradiation on bone regeneration in midpalatal suture during expansion in the rat. Am J Orthod Dentofacial Orthop 1997; 111: 525-532.

20. Kim SJ, Kang YG, Park JH, Kim EC, Park YG. Effects of lowintensity laser therapy on periodontal tissue remodeling during relapse and retention of orthodontically moved teeth. Lasers Med Sci 2013; 28: 325-333.

21. Franzen TJ, Zahra SE, El-Kadi A, Vandevska-Radunovic V. The influence of low-level laser on orthodontic relapse in rats. Eur J Orthod 2015; 37: 111-117.

22. Kim SJ, Chou MY, Park YG. Effect of low-level laser on the rate of tooth movement. Seminars in Orthodontics 2015; 21: 210-218.

23. Moslemzadeh SH, Sohrabi A, Rafighi A, Farshidnia S. Comparison of stability of the results of orthodontic treatment and gingival health between Hawley and vacuum-formed retainers. J Contemp Dent Pract 2018; 19: 443-449.

24. Varga S, Spalj S, Milosevic SA, et al. Changes of bite force and occlusal contacts in the retention phase of orthodontic treatment: a controlled clinical trial. Am J Orthod Dentofacial Orthop 2017; 152: 767-777.

25. O'Rourke N, Albeedh H, Sharma P, Johal A. Effectiveness of bonded and vacuum-formed retainers: a prospective randomized controlled clinical trial. Am J Orthod Dentofacial Orthop 2016; 150: 406-415.

26. Doshi-Mehta G, Bhad-Patil WA. Efficacy of low-intensity laser therapy in reducing treatment time and orthodontic pain: a clinical investigation. Am J Orthod Dentofacial Orthop 2012; 141: 289-297.

27. Sauget E, Covell DA, Boero RP, Lieber WS. Comparison of occlusal contacts with use of Hawley and clear overlay retainers. Angle Orthod 1997; 67: 223-230.

28. Forde K, Storey M, Littlewood SJ, Scott P, Luther F, Kang J. Bonded versus vacuum-formed retainers: a randomized controlled trial. Part 1: stability, retainer survival, and patient satisfaction outcomes after 12 months. Eur J Orthod 2018; 40: 387-398.

29. Kaya Y, Tunca M, Keskin S. Comparison of two retention appliances with respect to clinical effectiveness. Turkish J Orthod 2019; 32: 72 .

30. Walker M. No differences seen in outcomes between three different methods of orthodontic retention. Evid Based Dent 2013; 14: 81-82.

31. Lo Giudice A, Isola G, Rustico L, Ronsivalle V, Portelli M, Nucera R. The efficacy of retention appliances after fixed orthodontic treatment: a systematic review and meta-analysis. Applied Sci 2020; 10: 3107.

32. Hoybjerg AJ, Currier GF, Kadioglu O. Evaluation of 3 retention protocols using the American Board of Orthodontics cast and radiograph evaluation. Am J Orthod Dentofacial Orthop 2013; 144 16-22.

33. Durbin DS, Sadowsky C. Changes in tooth contacts following orthodontic treatment. Am J Orthod Dentofacial Orthop 1986; 90: 375-382.
34. Haydar B, Cier S, Saatçi P. Occlusal contact changes after the active phase of orthodontic treatment. Am J Orthod Dentofacial Orthop 1992; 102: 22-28.

35. Başçiftçi FA, Uysal T, Sari Z, Inan O. Occlusal contacts with different retention procedures in 1-year follow-up period. Am J Orthod Dentofacial Orthop 2007; 131: 357-362.

36. Greco PM, English JD, Briss BS, et al. Posttreatment tooth movement: for better or for worse. Am J Orthod Dentofacial Orthop 2010; 138: 552-558.

37. Nett BC, Huang GJ. Long-term posttreatment changes measured by the American Board of Orthodontics objective grading system. Am J Orthod Dentofacial Orthop 2005; 127: 444-450.

38. McDermott P, Millett D, Field D, Van Den Heuvel A, Erfida I. Lower incisor retention with fixed or vacuum-formed retainers. J Dent Res 2008; 87.

39. Xiao-Cen X, Ren-Mei L, Guo-Hua T. Clinical evaluation of lingual fixed retainer combined with Hawley retainer and vacuumformed retainer. Shanghai Kou Qiang Yi Xue 2011; 20: 623-626 [Article in Chinese]. 